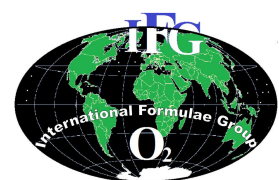

\title{
Inventaire ethno-pharmacologique des plantes utilisées dans le traitement des pathologies parasitaires dans les élevages extensifs et semi-intensifs du Bénin
}

\author{
C. A. OGNI ${ }^{1 *}$, M. T. KPODEKON ${ }^{1}$, H. G. DASSOU ${ }^{2}$, C. K. BOKO ${ }^{1}$, \\ B. G. KOUTINHOUIN ${ }^{1}$, J. T. DOUGNON ${ }^{1}$, A. K. I. YOUSSAO ${ }^{1}$, \\ H. YEDOMONHAN ${ }^{2}$ et A. AKOEGNINOU ${ }^{2}$. \\ ${ }^{1}$ Université d'Abomey-Calavi (UAC), Ecole Polytechnique d'Abomey-Calavi (EPAC), Département de \\ Production et Santé Animales, Laboratoire de Recherches en Biologie Appliquée (LARBA), \\ 01 BP 2009 Cotonou, Bénin. \\ ${ }^{2}$ Université d'Abomey-Calavi (UAC), Faculté des Sciences et Techniques (FAST), Département de Biologie \\ Végétale, Laboratoire de Botanique et Ecologie Végétale (LABEV), 01 BP 4521 Cotonou, Bénin. \\ *Auteur correspondant, E-mail : ogniabis@yahoo.fr
}

\section{RESUME}

L'Afrique regorge d'une importante réserve de plantes utilisées en ethnomédecine vétérinaire. La présente étude vise à répertorier une gamme de plantes médicinales habituellement utilisées pour traiter les pathologies parasitaires des animaux. A cet effet, une enquête ethno-botanique a été réalisée auprès de 787 éleveurs et agro-éleveurs des huit zones agro-écologiques du Bénin, de juin à décembre 2013. Au total, cinq (5) plantes médicinales (Azadirachta indica, Cajanus cajan, Cissus quadrangularis, Nicotiana tabacum et Vernonia amygdalina) sont utilisées pour traiter la coccidiose contre deux (2) plantes (Bombax costatum et Prosopis africana) pour la piroplasmose et douze (12) plantes dont A. polyacantha, C. sieberiana, C. febrifuga, D. microcarpum, P. kotschyi, Z. mays pour la trypanosomiase. En outre, dix-huit (18) plantes dont A. digitata, A. senegalensis, A. leiocarpa, Bambusa vulgaris, $F$. exasperata ont été inventoriées pour traiter les ectoparasitoses contre dix-sept (17) plantes dont A. flagellaris, C. papaya, E. guineensis, M. charantia, Senna alata pour la gale et quarante-deux (42) plantes dont A. digitata, A. sativum, Anacardium occidentale, A. leiocarpa, B. ferruginea, C. papaya, C. ambrosioides, K. senegalensis, $F$. sur, M. inermis, $N$. canensis pour traiter les helminthiases. Les organes les plus utilisés sont les feuilles et les écorces. La majorité des remèdes sont préparés sous forme de décoction. Des tests sur l'efficacité de ces plantes permettront de mieux les valoriser dans la lutte contre les pathologies parasitaires.

(C) 2014 International Formulae Group. All rights reserved.

Mots clés : Maladies parasitaires, phyto-pharmacologie, enquête, Bénin.

\section{INTRODUCTION}

L'élevage est l'une des principales activités entreprises par l'homme pour faire face au problème de sécurité alimentaire. Il contribue à l'économie mondiale en général et des pays africains en particulier. Au Bénin, une étude réalisée par Bongi et al. (2009) a révélé que le sous-secteur de l'élevage contribue au Produit Intérieur Brut à hauteur de 15 à $18 \%$. Malgré cette contribution, le 
déficit en protéines d'origine animale se pose dans l'alimentation des populations avec comme corolaire une augmentation des importations des produits carnés au fil des années (CountryStat, 2014). Dans le cadre de la relance de la filière agricole au Bénin, des efforts ont été fournis par le gouvernement pour augmenter le niveau de production des animaux tout en assurant une bonne prévention des pathologies dominantes fréquemment observées dans les exploitations (DE, 2011). Malgré ces dispositions, l'élevage est confronté à de sérieux problèmes d'ordre pathologique dont les pathologies bactériennes, virales, parasitaires et métaboliques. Parmi ces pathologies, les parasitoses occupent une place de choix en raison des pertes qu'elles occasionnent sur la productivité des animaux (Krecek et Waller, 2006 ; Chiejina et al., 2010). Les traitements de ces parasitoses sont le plus souvent réalisés avec des produits vétérinaires importés qui ne sont généralement pas à la portée des petits éleveurs (Bénoît, 2008). Aussi, leur qualité est-elle parfois douteuse, principalement à la faveur des mauvaises conditions de conservation et de transport. Vu l'importance de la pharmacopée vétérinaire dans les élevages traditionnels en Afrique (Jiofack et al., 2010) et particulièrement au Bénin, il apparaît évident de promouvoir ces alternatives endogènes de traitement ou de prévention de ces maladies par l'utilisation d'extraits de plantes. Selon Ngeh et al. (2007), l'usage de la pharmacopée a connu un regain d'intérêt en Afrique et constitue un début de solution aux problèmes de résistance des parasites aux molécules synthétiques. Ainsi, à l'instar des pays en développement au Sud du Sahara qui pratiquent la pharmacopée vétérinaire, la présente étude ethnopharmacologique se propose d'inventorier une gamme de plantes qui entrent dans les pratiques thérapeutiques endogènes appliquées aux maladies parasitaires dans les élevages du Bénin. Cette étude vise spécifiquement à déterminer la distribution des pathologies parasitaires par zone agro- écologique du Bénin; inventorier les plantes médicinales et les différentes recettes utilisées par les populations pour le traitement de ces pathologies.

\section{MATERIEL ET METHODES}

\section{Milieux d'enquête}

L'enquête ethno-pharmacologique a été effectuée dans les huit zones agro-écologiques du Bénin. Ce pays est situé entre $6^{\circ} 30^{\prime}$ et $12^{\circ} 30^{\prime}$ de Latitude Nord et, entre $1^{\circ}$ et $3^{\circ} 40^{\prime}$ de Longitude Est. Dans chaque zone, les Communes enquêtées (Figure 1) ont été celles qui possèdent un effectif en cheptel animal important et qui sont d'accès facile. Les zones agro-écologiques ont été subdivisées en tenant compte de leurs facteurs pédo-climatiques et des cultures agricoles qui y sont produites (MAEP, 2000). L'étude s'est déroulée de juin à décembre 2013.

La méthodologie utilisée est celle d'une enquête rétrospective sur les pathologies et les thérapeutiques traditionnelles utilisées par les pasteurs et agro-pasteurs pour les prévenir ou les traiter dès leur apparition. Un questionnaire d'enquête individuel a été utilisé. Il renseigne sur l'identité de l'informateur, les pathologies parasitaires dominantes rencontrées chez les animaux, leurs causes, les saisons d'apparition et sur les remèdes traditionnels à base de plantes utilisés pour les traiter (plantes, organes utilisés, modes de préparation, posologies et voies d'administration). Les pathologies dominantes ont été déterminées en fonction de leur fréquence de citation par les éleveurs. Cette fréquence est déterminée par la formule :

$$
F c=\frac{n}{N} \times 100
$$

Fc : fréquence de citation,

$\mathrm{n}$ : nombre de citation de la pathologie,

$\mathrm{N}$ : nombre total de citation des pathologies.

Le choix des éleveurs a été basé sur leur accessibilité et leur disponibilité à fournir des informations précises sur les soins traditionnels auxquels ils font recours pour 
traiter les maladies parasitaires dans leurs élevages. L'enquête a permis de collecter les informations auprès de 787 éleveurs constitués de $89,45 \%$ d'hommes et $10,55 \%$ de femmes diversement répartis dans les huit zones d'étude. A l'issue de l'enquête, des pathologies animales ont été identifiées en tenant compte de leurs descriptions faites par les éleveurs questionnés. De plus, les plantes médicinales et les recettes traditionnelles utilisées par les éleveurs pour le traitement de ces pathologies ont été inventoriées. Ces plantes ont été récoltées et identifiées in situ et certifiées par l'Herbier National du Bénin situé à l'Université d'Abomey-Calavi.

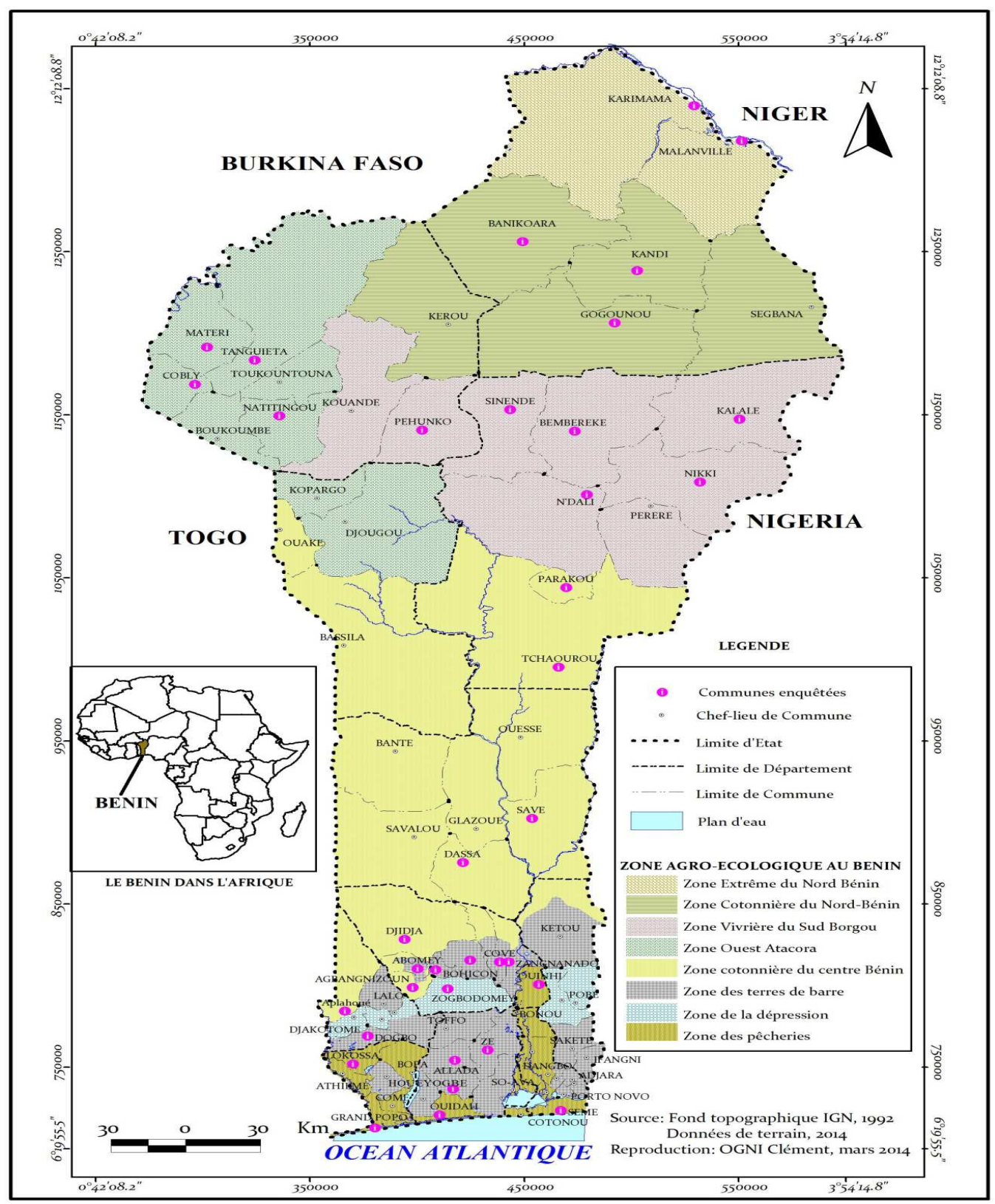

Figure 1: Représentation des zones agro-écologiques et des Communes enquêtées. 


\section{Analyses statistiques}

Les données collectées sur le terrain ont été codées et enregistrées dans une base de données conçue sur le Logiciel Excel. Elles ont été soumises à l'analyse dans le Logiciel SAS (Statistical Analysis System, 2006).

Les fréquences et les moyennes ont été calculées par zone agro-écologique. La procédure Proc freq a été utilisée pour déterminer la significativité de l'effet zone agro-écologique en utilisant le test de $\chi^{2}$ et les fréquences ont été comparées deux à deux par le test bilatéral de Z. Pour chaque fréquence relative, un intervalle de confiance (IC) à 95\% a été calculé suivant la formule :

$$
I C P=1,96 \sqrt{\frac{[P(1-P)]}{N}} \text { où } \mathrm{P} \text { est }
$$

la fréquence relative et $\mathrm{N}$ la taille de l'échantillon.

Un modèle linéaire à un seul facteur de variation (zone agro-écologique) a été utilisé pour l'Analyse de Variance. Le test de F a été utilisé pour déterminer la signification de l'effet zone agro-écologique et les moyennes ont été calculées et comparées par zone agroécologique par le test $\mathrm{t}$ de student.

La procédure Proc corresp a été utilisée pour l'analyse factorielle des correspondances (AFC). Les variables qui ont été prises en compte étaient: les pathologies parasitaires (coccidiose, ectoparasitose, gale, trypanosomiase, piroplasmose, helminthiase) et les plantes utilisées dans le traitement de ces pathologies. Une classification ascendante hiérarchique sur la base des coordonnées des pathologies et des plantes utilisées dans leur traitement sur les composantes de l'AFC les plus significatifs a été réalisée par la suite.

\section{RESULTATS}

\section{Caractéristiques socio-professionnelles des enquêtés}

Les individus interrogés dans l'extrême Nord-Bénin sont à $100 \%$ des éleveurs contre $83,16 \%$ dans la zone vivrière du Sud-Borgou ; $77,17 \%$ dans la zone des pêcheries et $36,7 \%$ dans la zone de dépression. Par contre, dans la zone Ouest-Atacora, les zones cotonnières du Nord et du Centre-Bénin et la zone des terres de barre, les enquêtés sont plus agro-éleveurs qu'éleveurs avec des proportions respectives de $85,71 \%, 57,97 \%, 58,27 \%$ et $44,14 \%$. Les proportions des éleveurs et agro-éleveurs ont varié significativement d'une zone agroécologique à une autre $(\mathrm{p}<0,001)$ (Tableau 1a).

Le niveau d'instruction des enquêtés a été diversifié dans les différentes zones agroécologiques. En effet, tous les enquêtés de l'extrême Nord du Bénin étaient non instruits. Dans la zone vivrière du Sud-Borgou, de l'Ouest-Atacora et les zones cotonnières du Nord et du Centre-Bénin, les individus enquêtés étaient non instruits avec des proportions respectives de $83,16 \%, 71,43 \%$, $94,2 \%$, et $80,31 \%$. Par contre, dans la zone des terres de barre et la zone de dépression, les personnes enquêtées étaient en majorité instruites. Elles avaient plus le niveau secondaire avec des proportions respectives de $45,05 \%$ et $37,61 \%$. De même, les personnes enquêtées dans la zone des pêcheries étaient en majorité instruites mais ayant plus le niveau primaire $(38,04 \%)$. Le niveau d'instruction des enquêtés a également varié significativement d'une zone agro-écologique à une autre $(\mathrm{p}<0,001)$ (Tableau $1 \mathrm{a})$. En ce qui concerne l'âge des enquêtés, les résultats ont révélé que les individus moins âgés se retrouvaient plus dans la zone de dépression (42,06 ans) et dans la zone des pêcheries (42,49 ans). Par contre, les plus âgés se retrouvaient dans la zone cotonnière du NordBénin avec une moyenne d'âge de 50,09 ans. Ensuite, la taille des ménages a été plus élevée dans l'extrême Nord-Bénin et dans la zone vivrière du Sud-Borgou avec respectivement 11,09 et 10,49 individus en moyenne. Par contre, les ménages de plus faibles effectifs sont rencontrés dans la zone de dépression et la zone des pêcheries. Ces effectifs ont été respectivement de 6,34 et 6,35 individus en moyenne avec des différences hautement significatives d'une zone agro-écologique à une autre $(\mathrm{p}<0,001)$ (Tableau 1b).

Tous les éleveurs de l'extrême NordBénin sont de l'ethnie peulh. Dans la zone cotonnière du Nord-Bénin, quatre ethnies ont été observées dont les peulh majoritaires à 
$88,41 \%$ sont suivis des bariba $(5,80 \%)$. En ce qui concerne la zone vivrière du Sud-Borgou, la proportion des peulh a été de $88,42 \%$ et de $8,42 \%$ pour les fon. Par contre, dans la zone Ouest-Atacora, l'ethnie gourmantché est plus élevée $(35,71 \%)$ suivie des peulh $(28,57 \%)$. La zone cotonnière du Centre-Bénin, la zone des terres de barre et des pêcheries ont présenté une diversité ethnique majoritairement répartie entre les peulh, les fon et assimilés.

\section{Typologie des pathologies soupçonnées}

Les pathologies parasitaires révélées par l'enquête ont été au nombre de six. Il s'agit de la coccidiose chez les poulets et les lapins, des ectoparasitoses et des helminthiases chez toutes les espèces; de la gale, de la trypanosomiase et de la piroplasmose chez les ruminants. Par rapport aux zones agro- écologiques, la coccidiose a été plus signalée $(\mathrm{p}<0,05)$ dans la zone des terres de barre, la zone de dépression et celle des pêcheries avec les fréquences respectives de $16,22 \% ; 12,84 \%$ et $13,04 \%$. Dans les zones cotonnières du Nord-Bénin et du Centre-Bénin, elle a été rarement rapportée par respectivement 1,45 et $0,79 \%$ des enquêtés. Par contre, cette pathologie n'a pas été signalée dans l'extrême Nord-Bénin, la zone vivrière du Nord-Bénin et dans la zone Ouest-Atacora. Quant aux ectoparasitoses, elles ont été plus rapportées $(\mathrm{p}<0,05)$ dans la zone vivrière du Nord-Bénin à une fréquence de $21,05 \%$ contre respectivement $4,44 \%$; $7,25 \% ; 4,72 \% ; 3,60 \% ; 8,26 \%$ et $9,78 \%$ dans l'extrême Nord, les zones cotonnières du Nord et du Centre, la zone des terres de barre, de dépression et des pêcheries. Cependant, elles n'ont pas été signalées dans la zone OuestAtacora. La gale a été plus signalée $(\mathrm{p}<0,05)$ par 40,22\% des enquêtés dans la zone des pêcheries que dans les zones cotonnières du Nord et du Centre, la zone vivrière du NordBénin, la zone Ouest-Atacora, des terres de barre et de dépression avec des proportions respectives de $2,9 \% ; 25,98 \% ; 1,05 \%$; $17,86 \% ; 22,07 \%$ et $23,85 \%$. Par contre, elles n'ont pas été signalées dans l'extrême Nord. Les helminthiases ont été rencontrées dans toutes les zones agro-écologiques du Bénin. En effet, elles ont été plus rapportées $(\mathrm{p}<0,05)$ dans la zone Ouest-Atacora par 78,57\% des enquêtés et moins signalées dans la zone des pêcheries $(36,96 \%)$. Elles ont, par ailleurs, été signalées dans l'extrême Nord, les zones cotonnières du Nord et du Centre, la zone vivrière du Nord-Bénin, de dépression et des pêcheries à des proportions respectives de $62,22 \%$; 56,52\% et $57,48 \%$; 64,21\% ; $57,21 \%$ et $53,21 \%$ des enquêtés. Par rapport à la piroplasmose, elle n'a été rapportée que dans zone cotonnière du Nord-Bénin par $4,35 \%$ des enquêtés. Quant à la trypanosomiase, elle n'a pas été signalée dans la zone des pêcheries. Par contre, elle a été plus signalée $(\mathrm{p}<0,05)$ dans l'extrême NordBénin par 33,33\% des enquêtés que dans les zones cotonnières du Nord et du Centre, la zone vivrière du Nord-Bénin, la zone OuestAtacora, des terres de barre et de dépression par respectivement 27,54\%; $11,02 \%$; $13,68 \% ; 3,57 \% ; 0,90 \%$ et $1,83 \%$ des enquêtés. Les proportions de cette pathologie ont significativement varié d'une zone à une autre (Tableau 2).

\section{Caractéristiques ethno-pharmacologiques}

Tous les éleveurs de l'extrême Nord, de la zone vivrière du Sud-Borgou, de la zone Ouest-Atacora, de la zone cotonnière du Centre et de la zone des pêcheries traitent ou préviennent les pathologies dans leurs élevages en utilisant les organes de plantes. Toutefois, dans les zones cotonnières du Nord-Bénin, des terres de barre et de la dépression, certains éleveurs n'apportent aucun soin sanitaire à leurs animaux dans les proportions respectives de $1,45 \% ; 0,45 \%$ et $1,83 \%$ des enquêtés. Trois types de pratiques thérapeutiques sont généralement utilisés par les éleveurs. Il s'agit des pratiques traditionnelles, modernes et mixtes. Les éleveurs ont tendance à faire plus usage de la médecine vétérinaire moderne $(47,55 \%)$ ou du traitement mixte $(45,70 \%)$. Néanmoins, quelques éleveurs des zones vivrières du SudBorgou, cotonnière du Centre-Bénin, des terres de barre et de la zone de dépression observent strictement les pratiques 
traditionnelles dans des proportions respectives de $1,05 \%, 2,36 \%, 20,72 \%, 9,17 \%$ et $20,70 \%$.

Les plantes entrant dans la préparation des remèdes contre les pathologies animales sont utilisées seules à une fréquence de $85,75 \%$ selon les résultats de l'enquête.

\section{Situation floristique antiparasitaire, Organes des plantes utilisées et modes de préparation}

L'exploration floristique a permis de différencier 67 espèces végétales de plantes appartenant à 38 familles botaniques dont les plus représentées sont les LeguminosaeCaesalpinioideae $(8,96 \%)$; les Combretaceae, les Euphorbiaceae et les Rutaceae avec des proportions de $5,97 \%$ chacune.

Les principales parties prélevées sur les plantes pour traiter les pathologies sont les feuilles, les écorces et les graines. Ces organes étaient utilisés en moyenne à des proportions respectives de $49,53 \% ; 26,07 \%$ et $12,09 \%$ (Figure 2).

Les modes de préparation de plantes médicinales utilisés plus couramment pour traiter les pathologies parasitaires animales sont la décoction $(22,75 \%)$, la trituration $(22,27 \%)$, la macération $(14,69 \%)$ et la crudité $(23,70 \%)$.

Les formes d'emploi préconisées sont la tisane $(44,79 \%)$ et les feuilles entières $(21,09 \%)$. La voie orale est la plus utilisée pour l'administration des remèdes antiparasitaires avec un taux de $84,60 \%$ contre $13,03 \%$ pour les applications externes. Les doses de ces préparations sont variables et non maîtrisées par la quasi totalité des utilisateurs.

Distribution des plantes utilisées dans le traitement des pathologies parasitaires

Le Tableau 3 présente les valeurs propres et les proportions d'informations concentrées sur 5 axes. Avec les trois premiers axes, $66,81 \%$ des informations de départ ont été contrôlées, garantissant une bonne précision d'interprétation. Ces axes ont été retenus pour l'interprétation des résultats de l'analyse factorielle des correspondances $\left(\chi^{2}=55\right)$. En ce qui concerne la contribution absolue des différentes modalités sur chacun des axes, l'ectoparasitose et la trypanosomiase sont bien présentées sur l'axe 1 avec des contributions respectives de 16,35 et $37,14 \%$ des variations. Les helminthiases et la piroplasmose sont bien représentées dans l'axe 2 avec des proportions respectives de 11,61 et $77,49 \%$ des variations, pendant que la coccidiose et la gale sont bien présentées dans l'axe 3 avec des proportions respectives de 63,65 et $18,48 \%$ des variations. Accessoirement, la piroplasmose est représentée dans l'axe 1 avec $15,57 \%$ des variations.

Le premier axe factoriel oppose les groupes de plantes Ntaba, Y, M3, D6, R6, E2, T2, X3 et J4 aux groupes P6 et G5 (Figure 3). L'axe 2 oppose Bcosta et Pafr au groupe de plantes I24 (Figure 3). Une classification ascendante hiérarchique réalisée sur les coordonnées de ces deux axes a permis de regrouper les pathologies parasitaires et les plantes utilisées pour les traiter en 6 groupes (Figure 3)

Le groupe 1 rassemble Bombax costatum et Prosopis africana pour traiter la piroplasmose. De ces deux plantes, Prosopis africana a été également utilisée dans la préparation des remèdes pour le traitement de la trypanosomiase et des helminthiases.

Le groupe 2 rassemble Prosopis africana, Acacia polyacantha, Combretum collinum, Crossopteryx febrifuga, Kigelia africana, Zea mays, puis Afzelia africana, Cassia sieberiana, Detarium microcarpum, Khaya senegalensis, Pseudocedrela kotschyi et Ximenia americana pour le traitement de la trypanosomiase.

Dans le groupe 3, Prosopis africana, Azadirachta indica, Vernonia amygdalina, Mitragyna inermis, Crateva adansonii, Anogeissus leiocarpa, Adansonia digitata, Allium sativum, Amaranthus spinosus, Anacardium occidentale, Bridelia ferruginea, Chenopodium ambrosioides, Citrus sinensis, Cochlospermum planchoni, Euphorbia kamerunica, Ficus sur, Ipomoea asarifolia, Isoberlinia doka, Mallotus oppositifolius, Mangifera indica, Moringa oleifera, Nelsonia canescens, Newbouldia laevis, Pleiocarpa 
pycnantha, Psidium guajava, Pteleopsis suberosa, Rhigiocarya racemifera, Sarcocephalus latifolius, Tamarindus indica, Terminalia macroptera et Zanthoxylum zanthoxyloides pour le traitement des helminthiases. Parmi ces plantes, Azadirachta indica et Vernonia amygdalina ont été également utilisées pour le traitement de la coccidiose, de la gale et des ectoparasitoses. De même, Mitragyna inermis, Crateva adansonii, Anogeissus leiocarpa et Adansonia digitata ont été utilisées pour les ectoparasitoses.

Quant au groupe 4, il comprend Cajanus cajan, Cissus quadrangularis, Azadirachta indica, Vernonia amygdalina et Nicotiana tabacum pour le traitement de la coccidiose. Outre le traitement de la coccidiose, Nicotiana tabacum a été aussi utilisée contre la gale.

Le groupe 5 prend en compte Nicotiana tabacum, Azadirachta indica, Vernonia amygdalina, Hyptis suaveolens, Ficus exasperata, Parkia biglobosa, Vitellaria paradoxa, Asparagus flagellaris, Ceratotheca sesamoides, Elaeis guineensis, Launaea taraxacifolia, Ocimum gratissimum, Tridax Procumbens, Annona senegalensis, Bambusa vulgaris, Clausena anisata, Jatropha curcas, Olax subscorpioidea, Vitex doniana, Momordica charantia et Senna alata pour traiter la gale. Parmi ces plantes, Momordica charantia et Senna alata ont été aussi utilisées contre les ectoparasitoses.

Le groupe 6 rassemble Annona senegalensis, Bambusa vulgaris, Clausena anisata, Jatropha curcas, Olax subscorpioidea, Vitex doniana, Momordica charantia, Senna alata, Carica papaya, Citrus aurantifolia, Spondias mombin, Mitragyna inermis, Crateva adansonii, Anogeissus leiocarpa, Nicotiana tabacum et Adansonia digitata pour le traitement des ectoparasitoses.

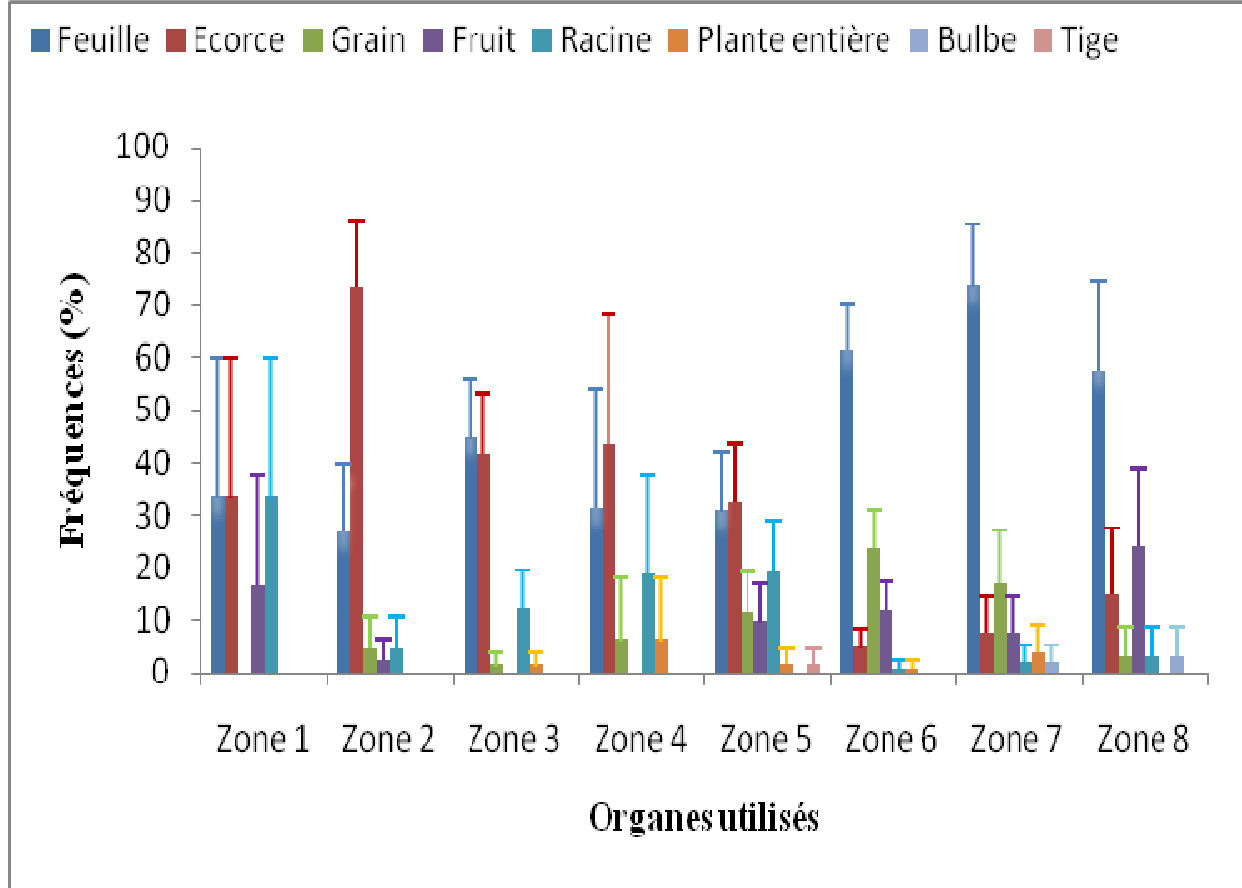

Figure 2: Pourcentages d'utilisation des organes de plantes pour le traitement des pathologies parasitaires animales au Bénin. Zone 1= extrême Nord-Bénin ; Zone 2= zone cotonnière du Nord-Bénin : Zone 3= zone vivrière du Sud-Borgou ; Zone 4= zone Ouest-Atacora ; Zone 5= zone cotonnière du Centre-Bénin ; Zone 6= zone des terres de barre ; Zone $7=$ zone de dépression ; Zone $8=$ zone des pêcheries. 
Tableau 1a : Caractéristiques socio-professionnelles des enquêtés.

\begin{tabular}{|c|c|c|c|c|c|c|c|c|c|c|}
\hline Variables & & $\begin{array}{c}\text { Zone1 } \\
(\mathrm{N}=45)\end{array}$ & $\begin{array}{r}\text { Zone2 } \\
(\mathrm{N}=69)\end{array}$ & $\begin{array}{c}\text { Zone3 } \\
(\mathrm{N}=95)\end{array}$ & $\begin{array}{c}\text { Zone4 } \\
(\mathrm{N}=28)\end{array}$ & $\begin{array}{c}\text { Zone5 } \\
(\mathrm{N}=127)\end{array}$ & $\begin{array}{c}\text { Zone6 } \\
(\mathrm{N}=\mathbf{2 2 2})\end{array}$ & $\begin{array}{c}\text { Zone7 } \\
(\mathrm{N}=109)\end{array}$ & $\begin{array}{c}\text { Zone8 } \\
(\mathrm{N}=92)\end{array}$ & ANOVA \\
\hline \multirow{6}{*}{$\begin{array}{l}\text { Profession des } \\
\text { enquêtés }\end{array}$} & Eleveur (\%) & $100 \mathrm{a}$ & $28,99 \mathrm{e}$ & $83,16 b$ & $7,14 \mathrm{f}$ & $27,56 \mathrm{e}$ & $38,29 \mathrm{~d}$ & $36,70 \mathrm{~d}$ & $77,17 \mathrm{c}$ & $* * *$ \\
\hline & Agriculteur (\%) & Od & Od & $1,05 \mathrm{c}$ & Od & $6,30 \mathrm{e}$ & $12,61 \mathrm{a}$ & $4,59 \mathrm{~b}$ & $6,52 b$ & $* * *$ \\
\hline & Commerçant $(\%)$ & $\mathrm{Ob}$ & $\mathrm{Ob}$ & $\mathrm{Ob}$ & $3,57 \mathrm{a}$ & $2,36 \mathrm{a}$ & $3,15 \mathrm{a}$ & $3,67 \mathrm{a}$ & $\mathrm{Ob}$ & $* * *$ \\
\hline & Vétérinaire (\%) & Od & Od & Od & $3,57 \mathrm{ab}$ & $1,57 \mathrm{c}$ & Od & $5,50 \mathrm{a}$ & $3,26 b c$ & $* * *$ \\
\hline & Agro-éleveur (\%) & of & $57,97 \mathrm{~b}$ & $12,63 \mathrm{e}$ & $85,71 \mathrm{a}$ & $58,27 \mathrm{~b}$ & $44,14 \mathrm{~d}$ & $30,28 \mathrm{~d}$ & $11,96 \mathrm{e}$ & $* * *$ \\
\hline & Autres $(\%)$ & of & $13,04 \mathrm{~b}$ & $3,16 \mathrm{~cd}$ & $0 \mathrm{c}$ & $3,94 \mathrm{c}$ & $1,80 \mathrm{de}$ & $19,27 \mathrm{a}$ & $1,09 \mathrm{e}$ & $* * *$ \\
\hline \multirow{4}{*}{$\begin{array}{l}\text { Niveau d'instruction } \\
\text { des enquêtés }\end{array}$} & Non instruit (\%) & $100 \mathrm{a}$ & $94,20 \mathrm{~b}$ & $83,16 c$ & $71,43 \mathrm{~d}$ & $80,31 \mathrm{c}$ & $26,13 \mathrm{e}$ & $28,44 \mathrm{e}$ & $22,83 \mathrm{e}$ & $* * *$ \\
\hline & Niveau primaire (\%) & of & Of & $6,32 \mathrm{e}$ & $14,29 \mathrm{c}$ & $8,66 \mathrm{~d}$ & $27,93 b$ & $22,02 b$ & $38,04 \mathrm{a}$ & $* * *$ \\
\hline & Niveau secondaire $(\%)$ & $0 \mathrm{e}$ & $2,90 \mathrm{~d}$ & $10,53 \mathrm{c}$ & $14,29 \mathrm{c}$ & $10,24 \mathrm{c}$ & $45,05 \mathrm{a}$ & $37,61 \mathrm{~b}$ & $33,70 \mathrm{~b}$ & $* * *$ \\
\hline & Niveau universitaire (\%) & Od & $2,90 \mathrm{~b}$ & $0 \mathrm{~d}$ & $0 \mathrm{~d}$ & $0,79 \mathrm{c}$ & $0 \mathrm{~d}$ & $9,17 \mathrm{a}$ & $2,17 b c$ & $* * *$ \\
\hline
\end{tabular}

Zone1 = extrême Nord-Bénin ; Zone2= zone cotonnière du Nord-Bénin : Zone3= zone vivrière du Sud-Borgou ; Zone4= zone Ouest-Atacora ; Zone5= zone cotonnière du Centre-Bénin ;

Zone6= zone des terres de barre ; Zone7= zone de dépression ; Zone8= zone des pêcheries ; N= nombre d'individus enquêtés.

Tableau 1b : Age et taille des ménages des enquêtés.

\begin{tabular}{lcccccccccc}
\hline Variables & $\begin{array}{c}\text { Zone1 } \\
(\mathbf{N = 4 5})\end{array}$ & $\begin{array}{c}\text { Zone2 } \\
(\mathbf{N = 6 9})\end{array}$ & $\begin{array}{c}\text { Zone3 } \\
(\mathbf{N = 9 5})\end{array}$ & $\begin{array}{c}\text { Zone4 } \\
(\mathbf{N}=\mathbf{2 8})\end{array}$ & $\begin{array}{c}\text { Zone5 } \\
(\mathbf{N}=\mathbf{1 2 7})\end{array}$ & $\begin{array}{c}\text { Zone6 } \\
(\mathbf{N}=\mathbf{2 2 2})\end{array}$ & $\begin{array}{c}\text { Zone7 } \\
(\mathbf{N}=\mathbf{1 0 9})\end{array}$ & $\begin{array}{c}\text { Zone8 } \\
(\mathbf{N}=\mathbf{9 2})\end{array}$ & DSR & Test \\
\hline Age (ans) & $46,91 \mathrm{ab}$ & $50,09 \mathrm{a}$ & $46,52 \mathrm{ab}$ & $47,68 \mathrm{ab}$ & $46,69 \mathrm{ab}$ & $45,79 \mathrm{~b}$ & $42,06 \mathrm{c}$ & $42,49 \mathrm{c}$ & 12,52 & $* * *$ \\
Ménage (personne) & $11,09 \mathrm{a}$ & $8,96 \mathrm{bc}$ & $10,49 \mathrm{a}$ & $9,71 \mathrm{ab}$ & $7,72 \mathrm{c}$ & $7,7 \mathrm{~cd}$ & $6,34 \mathrm{~d}$ & $6,35 \mathrm{~d}$ & 4,49 & $* * *$ \\
\hline
\end{tabular}

DSR : déviation standard résiduelle; ANOVA : test de significativité de l'analyse de variance ; Les moyennes de la même ligne suivis de lettres différentes, diffèrent significativement au seuil de 5\%.***: $\mathrm{p}<0,001 ;$ Zone1 = extrême Nord-Bénin ; Zone2= zone cotonnière du Nord-Bénin : Zone3= zone vivrière du Sud-Borgou ; Zone4= zone Ouest-Atacora : Zone $5=$ zone cotonnière du Centre-Bénin ; Zone6= zone des terres de barre ; Zone7= zone de dépression ; Zone $8=$ zone des pêcheries ; N= nombre d'individus enquêtés 
Tableau 2 : Répartition des pathologies parasitaires dans les zones agro-écologiques.

\begin{tabular}{|c|c|c|c|c|c|c|c|c|c|}
\hline Variables & $\begin{array}{c}\text { Zone1 } \\
(\mathrm{N}=45)\end{array}$ & $\begin{array}{c}\text { Zone2 } \\
(\mathrm{N}=69)\end{array}$ & $\begin{array}{c}\text { Zone3 } \\
(\mathrm{N}=95)\end{array}$ & $\begin{array}{c}\text { Zone4 } \\
(\mathrm{N}=28)\end{array}$ & $\begin{array}{c}\text { Zone5 } \\
(\mathrm{N}=127)\end{array}$ & $\begin{array}{c}\text { Zone6 } \\
(\mathrm{N}=222)\end{array}$ & $\begin{array}{c}\text { Zone7 } \\
(\mathrm{N}=109)\end{array}$ & $\begin{array}{c}\text { Zone8 } \\
(\mathrm{N}=92)\end{array}$ & ANOVA \\
\hline Coccidiose (\%) & $0 \mathrm{c}$ & $1,45 b$ & $0 \mathrm{c}$ & $0 \mathrm{c}$ & $0,79 b$ & $16,22 \mathrm{a}$ & $12,84 a$ & $13,04 \mathrm{a}$ & $* * *$ \\
\hline Ectoparasitose (\%) & $4,44 \mathrm{e}$ & $7,25 \mathrm{~cd}$ & $21,05 \mathrm{a}$ & Of & $4,72 \mathrm{de}$ & $3,6 \mathrm{e}$ & $8,26 \mathrm{bc}$ & $9,78 b$ & $* * *$ \\
\hline Gale $(\%)$ & Of & $2,9 \mathrm{~d}$ & $1,05 \mathrm{e}$ & $17,86 \mathrm{c}$ & $25,98 b$ & $22,07 b$ & $23,85 b$ & $40,22 \mathrm{a}$ & $* * *$ \\
\hline Helminthiase (\%) & $62,22 b$ & $56,52 \mathrm{c}$ & $64,21 b$ & $78,57 \mathrm{a}$ & $57,48 \mathrm{c}$ & $57,21 \mathrm{c}$ & $53,21 \mathrm{c}$ & $36,96 \mathrm{~d}$ & $* * *$ \\
\hline Piroplasmose $(\%)$ & $0 \mathrm{~b}$ & $4,35 \mathrm{a}$ & $0 \mathrm{~b}$ & $0 \mathrm{~b}$ & $\mathrm{Ob}$ & $0 \mathrm{~b}$ & $0 \mathrm{~b}$ & $0 \mathrm{~b}$ & $* * *$ \\
\hline Trypanosomiase (\%) & $33,33 a$ & $27,54 b$ & $13,68 \mathrm{c}$ & $3,57 \mathrm{~d}$ & $11,02 \mathrm{c}$ & $0,9 \mathrm{e}$ & $1,83 \mathrm{e}$ & Of & $* * *$ \\
\hline
\end{tabular}

ANOVA : test de significativité de l'analyse de variance ; Les pourcentages de la même ligne suivis de lettres différentes, diffèrent significativement au seuil de $5 \%$. $* * *: p<0,001$ Zone1= extrême Nord-Bénin ; Zone2= zone cotonnière du Nord-Bénin : Zone3= zone vivrière du Sud-Borgou ; Zone4= zone Ouest-Atacora; Zone5= zone cotonnière du Centre-Bénin ; Zone6= zone des terres de barre ; Zone7= zone de dépression ; Zone8= zone des pêcheries ; $\mathrm{N}=$ nombre de citation des pathologies parasitaires.

Tableau 3 : Valeurs propres et proportions d'informations concentrées sur les axes.

\begin{tabular}{lcccc}
\hline Valeur singulière & Inertie principale & $\boldsymbol{\chi}^{\mathbf{2}}$ & $\boldsymbol{\%}$ & \% cumulé \\
\hline 0,89 & 0,79 & 76,03 & 26,40 & 26,40 \\
0,80 & 0,64 & 61,39 & 21,31 & 47,71 \\
0,76 & 0,57 & 55,00 & 19,10 & 66,81 \\
0,73 & 0,53 & 51,28 & 17,80 & 84,61 \\
0,68 & 0,46 & 44,32 & 15,39 & 100,00 \\
Total & 3,00 & 288,02 & 100,00 & - \\
\hline
\end{tabular}




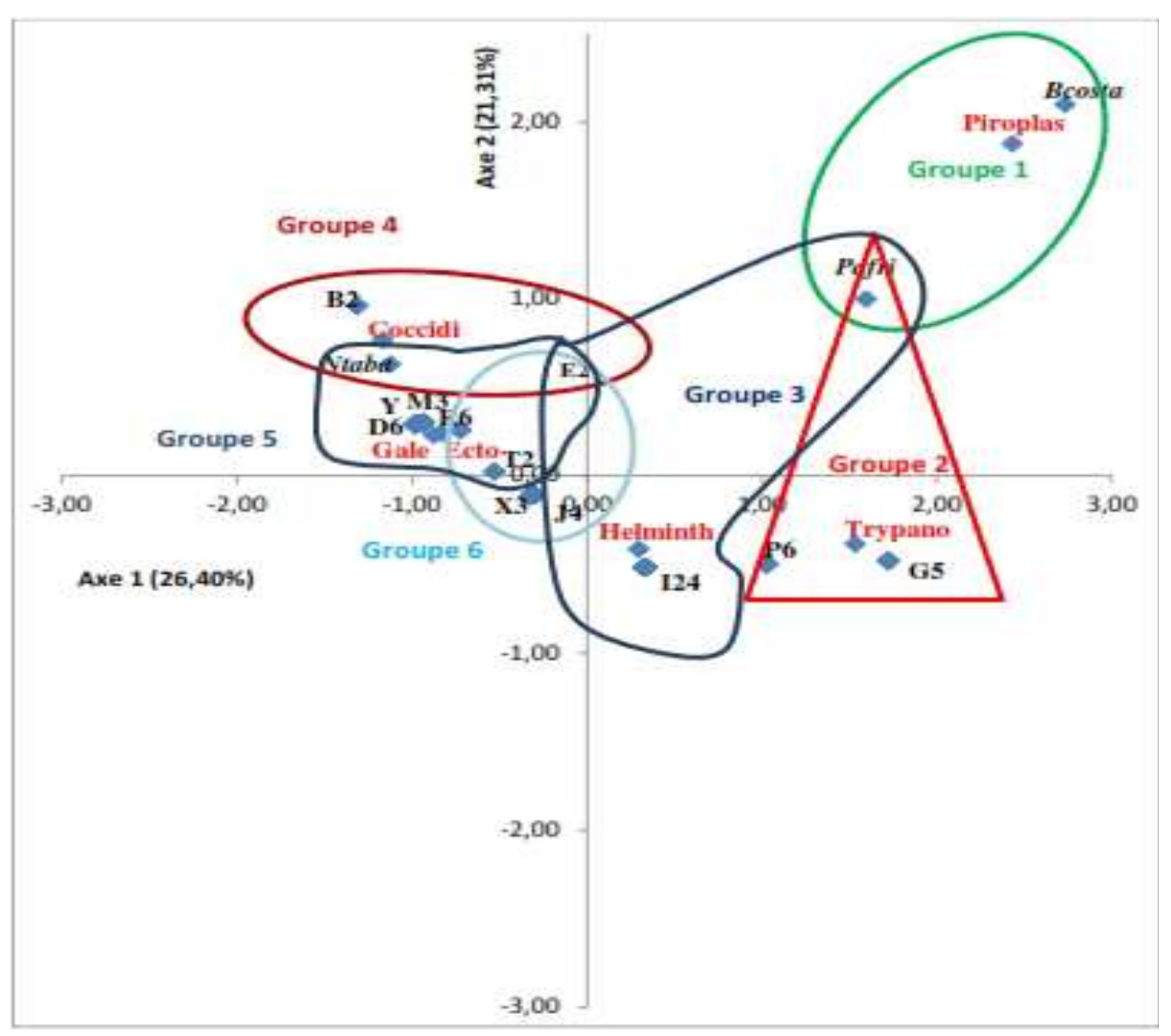

Figure 3 : Distribution des plantes en rapport avec les pathologies parasitaires animales sur le plan factoriel représentée par les deux premiers axes. Coccidi $=$ coccidiose Ecto $=$ ectoparasitose Trypano = trypanosomiase; Piroplas = piroplasmose; Helminth = helminthiase; J4 = (Mitragyna inermis, Crateva adansonii, Anogeissus leiocarpa, Adansonia digitata); D6 = (Asparagus flagellaris, Ceratotheca sesamoides, Elaeis guineensis, Launaea taraxacifolia, Ocimum gratissimum, Tridax Procumbens $) ; \mathbf{G 5}=($ Acacia polyacantha, Combretum collinum, Crossopteryx febrifuga, Kigelia africana, Zea mays $) ; \mathbf{E 2}=($ Azadirachta indica, Vernonia amygdalina $) ; \mathbf{T} 2=($ Momordica charantia, Senna alata $) ; \mathbf{B 2}=($ Cajanus cajan, Cissus quadrangularis $) ; \mathbf{X 3}=($ Carica papaya, Citrus aurantifolia, Spondias mombin $) ; \mathbf{M 3}=($ Ficus exasperata, Parkia biglobosa, Vitellaria paradoxa $) ; \mathbf{Y}=$ Hyptis suaveolens $;$ Bcost $=$ Bombax costatum $;$ Ntaba $=$ Nicotiana tabacum $;$ Pafri $=$ Prosopis africana $; \mathbf{P 6}=($ Afzelia africana, Cassia sieberiana, Detarium microcarpum, Khaya senegalensis, Pseudocedrela kotschyi, Ximenia americana $) ; \mathbf{R 6}=($ Annona senegalensis, Bambusa vulgaris, Clausena anisata, Jatropha curcas, Olax subscorpioidea, Vitex doniana $) ; \mathbf{I 2 4}=($ Allium sativum, Amaranthus spinosus, Anacardium occidentale, Bridelia ferruginea, Chenopodium ambrosioides, Citrus sinensis, Cochlospermum planchoni, Euphorbia kamerunica, Ficus sur, Ipomoea asarifolia, Isoberlinia doka, Mallotus oppositifolius, Mangifera indica, Moringa oleifera, Nelsonia canescens, Newbouldia laevis, Pleiocarpa pycnantha, Psidium guajava, Pteleopsis suberosa, Rhigiocarya racemifera, Sarcocephalus latifolius, Tamarindus indica, Terminalia macroptera, Zanthoxylum zanthoxyloides).

\section{DISCUSSION}

L'enquête ethno-pharmacologique des plantes utilisées pour traiter les pathologies parasitaires animales au Bénin a montré que les utilisateurs ont une connaissance plus large respectivement sur les espèces végétales efficaces dans le traitement des helminthiases, des ectoparasitoses, de la gale, de la trypanosomiase, de la coccidiose et de la piroplasmose.

Au cours de cette étude, quarante-deux (42) plantes étaient inventoriées pour traiter 
les helminthiases parmi lesquelles Khaya senegalensis, Chenopodium ambrosiodes, Carica papaya, Zantoxylum zantozyloides, Ximenia americana, Momordica charantia, Azadirachta indica, Mitragyna inermis, Sarcocephalus latifolius, Bridelia ferruginea, Anogeissus leiocarpa, etc. Dans une étude précédente, Hounzangbé-Adoté et al. (2001) avaient recensé cinq (Zanthoxylum zanthozylö̈des, Zea mays, Momordica charantia, Morindia lucida et Newbouldia laevis) au Sud-Bénin. Au cours de la présente étude, seules Zea mays et Morindia lucida n'ont pas été rapportées pour les cas d'helminthiase. Les travaux menés par Attindéhou et al. (2012) au Sud-Bénin avaient permis d'inventorier vingt et une (21) espèces. Parmi ces espèces, Ficus exasperata, Gliricidia sepium, Ocimum gratissimum, Phyllantus amarus, Ocimum canum, Jatropha curcas, Cassia italica, Petiveria alliacea, Harrisonia abyssinica, Trichilia prieuriana, Cajanus cajan et Ficus unibellata n'ont pas été rapportées par les enquêtés au cours de la présente étude. Cette disparité serait due à la méconnaissance ou l'absence de ces plantes dans leurs aires d'étude, notre travail étant réalisé sur tout le territoire national et compte tenue de l'action anthropique des populations sur les savanes. Au Burkina Faso, Kaboré et al. (2007) avaient identifié quinze (15) espèces pour traiter les parasitoses digestives des petits ruminants. Au-delà des plantes communes à ces différents travaux, la présente étude a ressorti vingt-huit nouvelles plantes dont Amaranthus spinosus, Anacardium occidentale, Bridelia ferruginea, Citrus sinensis, Cochlospermum planchonii, Euphorbia kameruniea, Ficus sur, Isoberlinia doka, Mallotus oppositifolius, Nelsonia canensis, Rhigyocarya racemifera, etc. Par ailleurs, des études de laboratoire ont montré que Anogeissus leiocarpa et Pseudocedrela kotschyi, ont des propriétés biologiques et chimiques efficaces dans le traitement ou la prévention d'infections gastro-intestinales (Koné et al., 2005). Au Cameroun, Tchoumboué et al. (1996) et Mpoame et Essomba (2000) ont traité avec succès des poulets contre des verminoses gastrointestinales avec de la poudre de Combretum $s p$. et avec les décoctions aqueuses de graines de Carica papaya. La confirmation de l'efficacité des extraits de certaines plantes inventoriées au cours des enquêtes vient conforter la connaissance empirique des activités des plantes par les éleveurs. Ceci pourrait s'expliquer par le fait que ces connaissances ont été acquises suite à des expériences répétées sur le terrain.

Pour les ectoparasitoses, dix-huit plantes médicinales avaient été citées par les individus enquêtés. Les travaux de Attindéhou et al. (2012), avaient permis d'inventorier deux plantes dans le Sud-Bénin dont Senna alata identifiée au cours du présent travail effectué dans toutes les zones agroécologiques. En dehors de cette plante, dixsept autres (Ficus exasperata, Olax subscorpioidea, Annona senegalensis, Adansonia digitata, Bambusa vulgaris, Clausena anisata, Crateva adansonii, etc.) ont davantage été recensées. Au Burkina Faso, Kaboré et al. (2012) avaient également recensé Nicotiana tabacum, Bambusa vulgaris, Azadirachta indic et Senna alata. Certaines plantes comme Annona senegalensis, Vitellaria paradoxa et Azadirachta indica sont encore utilisées et efficace dans le traitement des parasitoses internes. Ceci expliquerait l'utilisation d'une même plante pour traitement de plusieurs pathologies à la fois.

Pour la gale, dix-sept (17) plantes étaient recensées pour y faire face. Au nombre de ces plantes, Ceratotheca sesamoides, Hyptis suaveolens et Lactuca taraxacifolia ont été de nouvelles espèces citées par les éleveurs pour préparer les remèdes. Ces plantes seraient identifiées comme efficaces après des expérimentations en milieux réels par les éleveurs eux-mêmes.

Quant à la trypanosomiase, maladie préjudiciable à l'économie de l'élevage au Sud du Sahara, plusieurs plantes médicinales dont Khaya senegalensis, Pseudocedrela kotschyi, Acacia polyacantha et Cassia sieberiana sont utilisées traditionnellement pour son traitement. Ce résultat corrobore celui de Atawodi et al. (2003) qui ont rapporté au cours d'une enquête que les éleveurs peulhs utilisent aussi bien Khaya senegalensis, Prosopis africana, 
Pseudocedrela kotschyi que Afzelia africana pour traiter la trypanosomiase.

La coccidiose est l'une des pathologies parasitaires qui ralentissent la croissance des animaux et à l'origine d'importants dégâts en élevage. Les résultats de cette enquête ethno pharmacologique ont montré qu'au Bénin, pour $\mathrm{y}$ faire face traditionnellement, les éleveurs font usage d'un certain nombre de plantes médicinales parmi lesquelles, Cissus quadrangularis, Vernonia amygdalina et Azadirachta indica. Aucun autre travail n'avait été réalisé dans ce cadre au Bénin. Ceci pourrait expliquer le manque d'informations ethno-vétérinaires par rapport à cette pathologie. Des travaux menés par Dossou (2008) ont montré une efficacité du tourteau d'Azadirachta indica sur le développement des coccidies isolées de poulets au Sénégal. Par ailleurs, l'efficacité d'autres plantes a été vérifiée et approuvée par certains auteurs sur les coccidies. C'est le cas par exemple de Carica papaya (Tanyu, 2000) et Sarcocephalus latifolius (Fall, 2007) utilisées pour traiter les helminthiases selon les résultats de l'enquête.

Les résultats de cette étude ont révélé que les éleveurs et/ou agro-éleveurs sont les détenteurs des connaissances ethnovétérinaires comme l'ont rapporté Kaboré et al. (2012). Un nombre important d'entre eux font encore recours à cette pratique ancestrale comme mentionné par Hounzangbé-Adoté (2005) qui évalue leur présence à près de $40 \%$ au Bénin.

La plupart des plantes recensées interviennent dans le traitement de plusieurs pathologies à la fois. Annona senegalensis cité pour le traitement de la gale a été également mentionné au cours des travaux de Makumbelo et al. (2008) contre les infections cutanées. Selon Honzangbé-Adoté et al. (2001), Carica papaya utilisé contre les parasitoses externes, est aussi employé dans le traitement des parasitoses internes.

Les organes les plus utilisés sur les plantes recensées sont les feuilles et les écorces. L'accès facile à ces différents organes justifierait leur usage élevé dans la préparation des remèdes comme l'ont rapporté Salhi et al. (2010). A l'issue des résultats obtenus de l'enquête, les détenteurs des connaissances dans le domaine de l'ethnomédecine vétérinaire avaient un âge supérieur ou égal à 45 ans. Ces résultats sont en accord avec ceux trouvés par Guedje et al. (2008) et Mpondo et Dibong (2012).

\section{Conclusion}

La flore béninoise dispose d'une richesse diversifiée de plantes médicinales à efficacité antiparasitaire. Cette étude a permis d'apprécier le niveau de connaissances de l'usage des plantes par les éleveurs et agroéleveurs du Bénin pour traiter différentes pathologies parasitaires animales. Au total, 67 espèces végétales appartenant à 38 familles botaniques ont été recensées comme plantes antiparasitaires et intervenant dans la préparation de 91 recettes. Toutefois, l'épanouissement de l'élevage dépend non seulement de la maîtrise des pathologies parasitaires mais aussi de plusieurs autres catégories de pathologies dont, les pathologies bactériennes, virales, de reproduction, etc. Il importe donc d'évaluer le potentiel ethnobotanique béninois utilisé dans le traitement de ces dernières.

\section{REFERENCES}

Atawodi SE, Bulus T, Ibrahim S, Ameh DA, Nok AJ, Mamman M, Galadiman MJ. 2003. In vitro trypanocidial effect of methanolic extract of some Nigerian Savannah Plants. Afr. J. Biotechnol., 2(9): 317-321.

Attindéhou S, Houngnimassoun MA, Salifou S, Biaou FC. 2012. Inventorying of herbal remedies used to control small ruminant's parasites in Southern Benin. Int. Multidiscip. Res. J., 2(8) : 14-16.

Bénoît E. 2008. Les changements climatiques: vulnérabilité, impacts et adaptation dans le monde de la médecine traditionnelle au Burkina Faso. Vertigo la revue électronique en science de 
l'environnement $\quad \mathbf{8}(1), \quad$ http:/vertigo. revues.org/index 1467.html.

Bongi S, Obama G, Le Dain SA, Cossi A. 2009. Analyse globale de la vulnérabilité, de la sécurité alimentaire et de la nutrition en République du Bénin., PAM, 168 p.

Chiejina SN, Behnke JM, Musongong GA, Nnadi PA, Ngongeh LA. 2010. Resistance and resilience of West African Dwarf goats of the Nigerian savanna zone exposed to experimental escalating primary and challenge infections with Haemonchus contortus. Vet. Parasitol., 171: 81-90.

CountryStat. 2014. Base de données, consulté à l'adresse http://www.countrystat. org/ben.

DE. 2011. Rapport d'activités annuelles de la Direction de l'Elevage, Cotonou, Bénin, $28 \mathrm{p}$.

Dossou AD. 2008. Effet du tourteau de neem (Azadirachta indica A. Juss) sur les coccidioses aviaires. Thèse Méd. Vét. : Dakar, $112 \mathrm{p}$.

Fall M. 2007. Recherche de l'activité antiparasitaire de trois plantes de la pharmacopée traditionnelle sénégalaise :Aphania senegalensis(Juss.expoir) Radlk (Sapindaceae) Cassia italica (mill) Lam (Caesalpinacéae). Nauclea latifolia (Rubiaceae) Thèse : Pharmacie, EISMV, Dakar. 129 p.

Guedje NM, Mouamfon M, Bigombé LP, Abéga SC, Lejoly J. 2008. Impact de la gestion socio-économique et technique des forêts communautaires à l'échelle des économies familiales. Cas de Kompia et Kabilone (Est-Cameroun). In Governance et Environnement en Afrique Centrale : le Modèle Participatif en Question, Roulet PA, Assemaker P (eds). Musée Royal de l'Afrique Centrale : Tervuren Belgique ; 139-157.

Hounzangbé-Adoté SM. 2001. L'élevage face à la pharmacopée en médecine vétérinaire au sud du Bénin. Bul. Rec. Agr. Bénin, 33: 1-9.
Hounzangbé-Adoté SM. 2005. Propriétés anthelminthiques de quatre plantes tropicales testées in vitro et in vivo sur les nématodes gastro-intestinaux chez les petits ruminants Djallonkés. Thèse de Doctorat, Université d'Abomey-Calavi, Bénin, $240 \mathrm{p}$.

Jiofack T, Fokunang C, Guedje NM, Kemeuze $\mathrm{V}$, Fongnzossie E, Nkongmeneck BA, Mapongmetsem PM, Tsabang N. 2010. Ethnobotanical uses of medicinals plants of two ethnoecological regions of Cameroon. Int. J. Med. Med. Sci, 2(3): 60-79.

Kaboré A, Tamboura HH, Belem GAM, Traoré A. 2007. Traitements ethnovétérinaire des parasitoses digestives des petits ruminants dans le plateau central du Burkina Faso. Int. J. Biol. Chem. Sci. 1(3): 297-304.

Kaboré A, Traoré A, Pare S, Sawadogo BC, Kalkoumdo G, Tamboura HH, Belem AMG. 2012. Ethno-medecinal study of plants used in ectoparasites infections of ruminant livestock in sahelian region of Burkina Faso, West Africa. J. Nat. Prod. Plant Resour., 2(5) : 611-616.

Koné MW, Kamanzi AK. 2006. Inventaire ethnobotanique et évaluation de l'activité anthelminthique des plantes médicinales utilisées en Côte d'Ivoire contre les helminthiases intestinales. Pharm. Med. Trad. Afr., 14: 55-72.

Koné WM, Kamanzi AK, Traoré D, Betschart B. 2005. Anthelmintic activity of medecinal plants used in Northern Côte d'Ivoire against intestinal helminthiasis. Pharmaceut. Biol., 43(1) : 72-78.

Krecek RC, Waller PJ. 2006. Towards of implementation of the "basket of option" approach to helminth parasite control of livestock : Emphasis of the tropics / subtropics. Vet. Parasitol., 139: 270282.

MAEP. 2000. Schéma directeur du développement agricole et rural. Vol. 2 stratégies sous sectorielles. Projet 
SPPD/BEN/99/004/.PNUD/FAO.

MAEP. Cotonou, Bénin, $171 \mathrm{p}$.

Mpoame M, Essomba L. 2000. Essai de traitement contre des parasitoses gastrointestinales $\mathrm{du}$ poulet avec des décoctions aqueuses de graines de papaye (Carica papaya). Rev. Elev. Méd. Vét. Pays Trop., 53(1): 23-25.

Mpondo Mpondo E, Dibong SD. 2012. Traditional knowledge on medicinal plants use by ethnic communities in Douala, Cameroon. European J. Med. Plants, 2(2): 159-176.

Ngeh JT, Wanyama J, Nuwanyakpa M, Django S. 2007. L'ethnomédecine vétérinaire. Agrodok n ${ }^{\circ} 44$.

Organisation Mondiale de la Santé. 2003.

Directives OMS Sur Les Bonnes Pratiques de Récolte (BPAR) Relatives
Aux Plantes Médicinales. OMS : Genève.

Salhi S, Fadli M, Zidane L, Douira A. 2010. Etudes floristique et ethnobotanique des plantes médicinales de la ville de Kénitra (Maroc). Laza., 31: 133-146.

SAS. 2006. SAS/STAT User's guide, vers, 6, 4th ed, Cary, NC, USA, SAS Inst.

Tanyu N. 2000. Effect of some medicinal plants (Carica papaya,Spilanthus filicanlus,Lantana camara,Bryphylium pinnatum)on the sporulation of Eimeria tenella oocysts. Mémoire de fin de maîtrise en Biologie Animale, Université de Dschang (Cameroun), 78 p.

Tchoumboué J, Mpoame M, Akamba DGM. 1996. Essai comparé de traitement de nématodes de poulet au "Sodivermyl "Baird et a l'écorce de Combretum sp. (combretacée). Tropicultura, 14 (1): 4-5. 